\title{
Treatment with adipose stem cells in a patient with moderate Alzheimer's disease: case report
}

\author{
This article was published in the following Dove Press journal: \\ Journal of Neurorestoratology \\ 15 October 2015 \\ Number of times this article has been viewed
}

\section{Magda Tsolaki ${ }^{1,2}$ \\ Stelios Zygouris 1,3 \\ Vassilis Tsoutsikas ${ }^{2}$ \\ Doxakis Anestakis $2,4,5$ \\ George Koliakos ${ }^{6,7}$}

'Third Department of Neurology, Medical School, Aristotle University of Thessaloniki, Thessaloniki, Greece; ${ }^{2}$ Greek Association of Alzheimer's Disease and Related Disorders, Thessaloniki, Greece; ${ }^{3} \mathrm{CND}+$, ${ }^{4}$ Laboratory of Forensic Medicine and Toxicology, Medical School, Aristotle University of Thessaloniki, Thessaloniki, Greece; ${ }^{5}$ Laboratory of General Biology, Medical School, Aristotle University of Thessaloniki, Thessaloniki, Greece; ${ }^{6}$ Biohellenika Stem Cells Bank, Thessaloniki, Greece; ${ }^{7}$ Department of Biological Chemistry, Medical School, Aristotle University of Thessaloniki, Thessaloniki, Greece
Correspondence: Doxakis Anestakis Laboratory of Forensic Medicine and Toxicology, Medical School, Aristotle University of Thessaloniki, Thessaloniki 54124, Greece Tel +30 6972697072 $\mathrm{Fax}+302310999686$

Email anestaki@auth.gr
Objective: This article presents the case of a female patient with Alzheimer's disease (AD). The patient was treated with cholinesterase inhibitors and also with intravenous administration of autologous adipose stem cells.

Methods: The patient was assessed with a neuropsychological battery including measures of general cognition, functional problems, neuropsychiatric issues, memory (verbal, visual and episodic), verbal learning and visuospatial abilities. Magnetic resonance imaging (MRI) scans were conducted before and after the treatment with stem cells.

Results: A transient and mild improvement of scores in measures of general cognition and neuropsychiatric issues was evident. A rapid deterioration followed the initial improvement. The first MRI scan showed ischemic areas in periventricular white matter of both hemispheres, as well as in both temporal and parietal lobes. The second MRI scan revealed the same picture with no significant changes.

Conclusion: This case report indicates that the administration of stem cells is feasible in a clinical setting however its effectiveness in the treatment of AD is uncertain. The improvement of the patient's condition highlights the potential therapeutic action of stem cells, however the rapid deterioration poses questions concerning limited effectiveness or possible side effects of stem cell administration. Further research is needed in order to clarify the method's effectiveness.

Keywords: Alzheimer's disease, stem cells, case report, dementia

\section{Introduction}

Alzheimer's disease (AD) is the most common form of dementia and affects more than 44 million people worldwide. With increased life expectancy, this number is expected to rise to more than 135 million people worldwide by $2050 .{ }^{1,2} \mathrm{AD}$ is characterized by progressive cognitive impairment and personality changes associated with degeneration and presence of neuritic plaques and neurofibrillary tangles. Therapeutic approaches include the implementation of cholinesterase inhibitors such as donepezil, rivastigmine, and galantamine, which present the major drug group, and memantine. Memory enhancers such as aniracetam, antioxidants such as vitamins E and C, gingko biloba, inhibitors of $N$-methyl-D-aspartate receptors, etc are not yet approved by the US Food and Drug Administration (FDA) for the treatment of AD. ${ }^{2,3}$

It has been reported that intravenous administration of autologous adipose-derived vascular fraction/adipose-derived stem cells (ADVF/ADSC) is a safe and convenient therapeutic modality, which according to preclinical evidence, can be beneficial for the treatment of AD. There is a meta-analysis about safety of cell therapy with mesenchymal stromal cells (SafeCell), including 34 studies with 1,012 patients; eight studies in the 
meta-analysis were randomized, with 321 participants total. No adverse short- or long-term effects were observed. ${ }^{3}$

The current report presents a 62-year-old female patient, with 8 years of formal education and a history of depression, suffering from moderate $\mathrm{AD}$, who had undergone treatment with adipose stem cells. Before the presentation of the patient, the method of preparation of stem cells that the patient was treated with is described.

\section{Method of preparation of stem cells}

ADVFs have been prepared from lipoaspirates using collagenase, as previously described., ${ }^{4,5}$ The patient underwent liposuction under general anesthesia in a sterile surgical operating-room environment. A plastic surgeon isolated 100-500 g of adipose tissue from the patient. Platelet-rich plasma was prepared after taking $50 \mathrm{~mL}$ of blood. Enzyme dissolution procedure of the adipose tissue was performed to separate the stromal cell fraction (pellet) from adipocytes. It was performed using collagenase type I solution under agitation for 2 hours, followed by two centrifugations at $100 \times g$ for 10 minutes the first time, and at $1,800 \times g$ for 10 minutes the second time. The final pellet was re-suspended, while a small volume of suspension fluid was used for flow cytometry analysis in a Coulter ${ }^{\circledR}$ Epics XL-MCL ${ }^{\mathrm{TM}}$. Bacterial check with the BacT/ALERT ${ }^{\circledR}$ system (with colorimetric carbon dioxide detector) was performed with $10 \mathrm{~mL}$ of the suspension. A volume of dimethyl sulfoxide (DMSO) solution was added in the remaining suspension so that the final volume contained $10 \%$ DMSO and 2\% HAES-steril ${ }^{\circledR} 200$, gradual cryopreservation of the cells followed, and they were then stored in air-tight cryovials sealed with CryoFlex ${ }^{\mathrm{TM}}$ membrane. These vials were stored at a constant temperature in liquid nitrogen. A total of $3-5 \times 10^{6}$ cells/g of adipose tissue were isolated. As ADVF represents a heterogeneous cell population, ${ }^{6}$ approximately $50 \%-70 \%$ of the total number of isolated cells were anticipated to be of mesenchymal origin, meaning CD29-, CD105-, CD90-, and CD73-positive, and CD34- and CD45-negative cells. In addition, $20 \%-30 \%$ of the total number of isolated cells were anticipated to be mature endothelial (CD31positive) and hematopoietic (CD34-positive) cells.

\section{Consent}

Written informed consent was obtained from the patient and her caregiver for publication of this case report and accompanying images. A copy of the written consent is available for review by the journal's Editor-in-Chief.

The study was performed according to Declaration of Helsinki requirements. The Ethical Committee of Aristotle University of Thessaloniki approved the protocol.

\section{Patient information}

A 62-year-old Caucasian female patient (education, 8 years) with a history of depression was examined at the Alzheimer's Day Care Unit "Saint Helen" for the first time on April 21, 2010.

\section{Personal past history}

Emotion problems first appeared 13 years before the patient's first visit, in 1997, when her mother passed away. A major depressive disorder appeared 7 years prior to first visit, along with memory impairment and problems with activities of daily living (ADL), when the patient faced issues with her relatives. In 2002, she underwent surgery to her foot performed with general anesthesia, and a similar operation was performed in May 2007. Since the surgery, her cognitive status started deteriorating. In the same year, she suffered major family stress (her grandson had an accident), which heavily contributed to both memory and emotional deterioration. The patient had had two abortions in the past.

\section{Current history}

\section{First visit:April 2I, 2010}

At her first visit, the patient complained mostly of memory impairment. She mentioned that she remembered almost nothing, and furthermore, she faced problems in expressive speech and repeated the same questions over and over. Eventually, she was not able to cope with daily activities as she had done in the past.

During the visit, she seemed to face problems with walking and stability, as well as with executing more complicated tasks. The neuropsychological examination scores were: Mini Mental State Examination (MMSE), 12/30; Neuropsychiatric Inventory (NPI), 8; Functional Rating Scale for Symptoms of Dementia (FRSSD), 20; Geriatric Depression Scale (GDS), 1 ; Functional Cognitive Assessment Scale (FUCAS), 99 points. Moreover, she performed the Rey Auditory Verbal Learning Test (RAVLT), in which she scored I $\rightarrow 2$, I-V $\rightarrow+1$, $\mathrm{VI} \rightarrow-3$; the Rey-Osterrieth Complex Figure Test (ROCFT), in which she scored 2.5; the Rivermead Behavioural Memory Test (RBMT), in which she scored 3 points; the Boston Naming Test (BNT), in which she scored 12-15-21, with a time of 8 minutes and 20 seconds She had been taking fluoxetine (Ladose) since 2004; she had also been taking gingko biloba 1 tablet $3 \times$ a day, memantine $\left(\right.$ Ebixa $\left.^{\circledR}\right) 10 \mathrm{mg} 1$ tablet $2 \times$ a day, and donepezil (Aricept) $10 \mathrm{mg} 1$ tablet $1 \times$ a day since 2007 . At her first visit, we stopped fluoxetine and escitalopram (Entact) 1 tablet $1 \times$ a day, was added to her medication regime.

On May 7, 2010, a brain magnetic resonance imaging (MRI) with gadolinium was performed and showed ischemic 


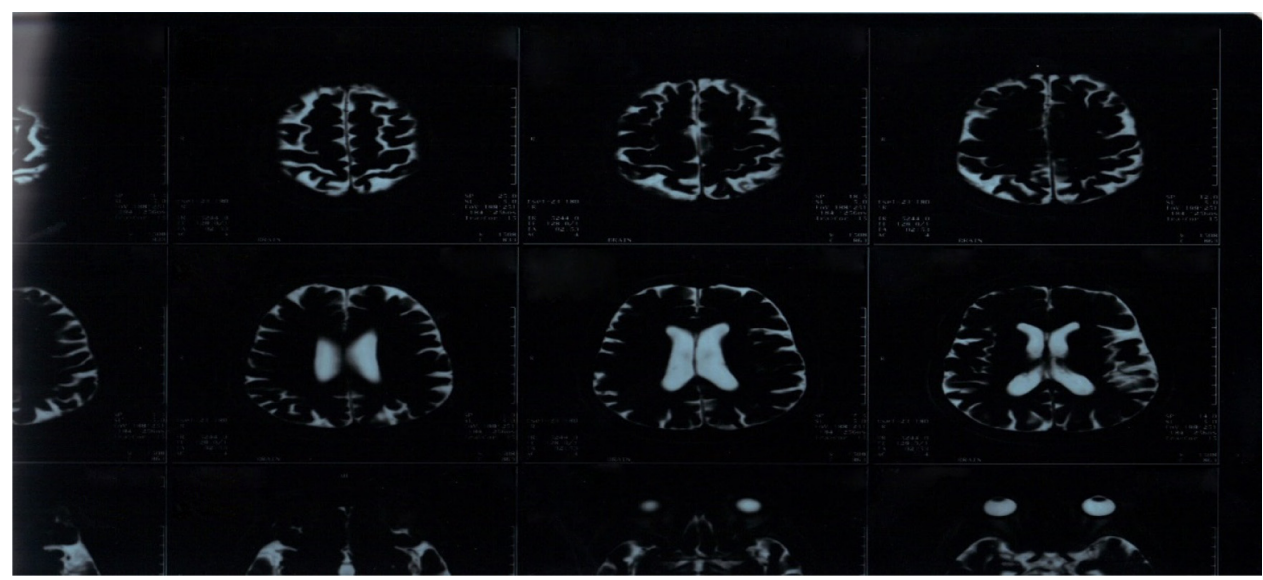

Figure I MRI T2W2 on May 7, 2010 showing enlargement in ventricles.

Abbreviation: MRI, magnetic resonance imaging.

areas in periventricular white matter of both hemispheres, and in both temporal and parietal lobes (Figures 1 and 2).

\section{Second visit: September 29, 2010}

On her second visit on September 29, 2010, the patient's condition appeared severely deteriorated. According to her spouse, she had been crying several times and appeared non-cooperative, resistant to receiving help from others, irritable and with lack of patience, especially when she was not able to perform simple tasks. Furthermore, she faced problems in spatial and time orientation, along with instability. The neuropsychological examination showed: MMSE, 9; NPI, 21; FRSSD, 22; GDS, 9; and FUCAS, 96 points. Escitalopram remained prescribed at $20 \mathrm{mg} 1 \times 1$. A rivastigmine patch at $9.5 \mathrm{mg}$ was added to her medications, as well as memantine $2 \times 1$ and gingko biloba $3 \times 1$. Finally, she was treated with folic acid $15 \mathrm{mg}, 1 \times 1$. Apolipoprotein $\mathrm{E}$ was $\varepsilon 3 / \varepsilon e 4$.

On September 30, 2010, $10 \mathrm{~mL}$ of autologous adipose stem cells $\left(31.6 \times 10^{6}\right.$ viable cells and $6.6 \times 10^{6}$ mesenchymal cells) was isolated from the patient, and a $5 \mathrm{~mL}$ solution of the cells $\left(9.6 \times 10^{6}\right.$ viable cells and $2 \times 10^{6}$ mesenchymal cells $)$ was administered intravenously on the same day.

\section{Third visit: November 2, 2010}

One month later, the patient's neuropsychological examination on November 2, 2010, 4 days after the stem cell transplantation, showed a slight improvement mainly in MMSE $=12$, in behavioral problems with NPI: 15 , and in ADL, FRSSD: 21. Her relatives were very happy and asked for a second intravenous administration of autologous adipose stem cells.

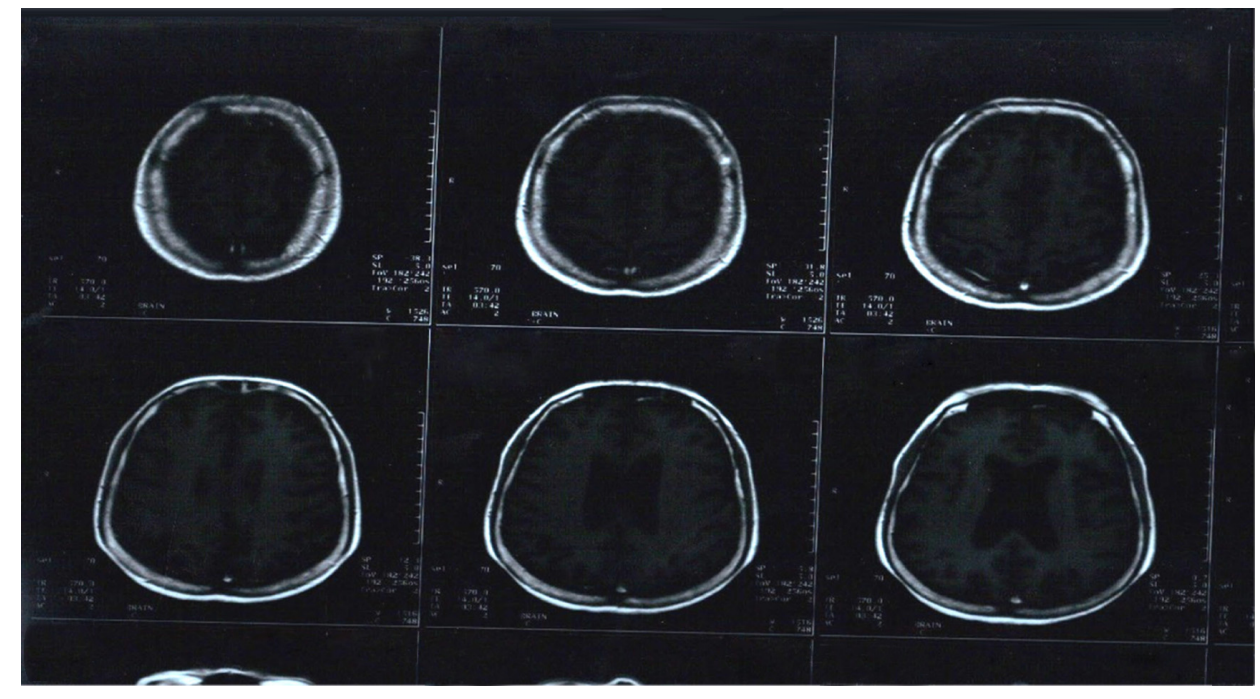

Figure 2 MRI TIWI on May 7, 2010 showing the ischemic areas in periventricular white matter, as well as atrophy of temporal and parietal lobes. Abbreviation: MRI, magnetic resonance imaging. 
Table I Neuropsychological assessment of the patient throughout the experimental stem cell therapy

\begin{tabular}{llllll}
\hline & MMSE & FRSSD & FUCAS & NPI & GDS \\
\hline First visit & 12 & 20 & 99 & 8 & I \\
Second visit & 9 & 22 & 96 & 21 & 9 \\
Third visit & 12 & 21 & No assessment & 15 & No assessment \\
Fourth visit & 6 & 27 & No assessment & 20 & No assessment \\
\hline
\end{tabular}

Abbreviations: MMSE, Mini Mental State Examination; NPI, Neuropsychiatric Inventory; FRSSD, Functional Rating Scale for Symptoms of Dementia; GDS, Geriatric Depression Scale; FUCAS, Functional Cognitive Assessment Scale.

\section{Fourth visit: May 4, 20I I}

The patient's cognitive status, functional ability as well as her depressive symptoms suffered a big deterioration, especially over the past month. According to her family, she was unable to complete a simple sentence and presented great difficulty in movement. Moreover, she suffered from hallucinations as she occasionally talked to people who were not there. The patient became insecure, very nervous and developed repetitive movements, for example, she played with her hair and scrubbed her hands. Finally, she became incontinent.

The neuropsychological examination showed: MMSE: 6, NPI: 20, FRSSD: 27, Hamilton: 15. Rivastigmine dose remained the same, whereas memantine changed to $10 \mathrm{mg}$ $1 \times 2$. Escitalopram was gradually stopped and replaced by Sertraline $100 \mathrm{mg}$, initially $1 / 4 \times 1$ and eventually $1 / 2 \times 1$. Hydroxyzine was added for coping with patient's irritability and Solifenacin $10 \mathrm{mg} 1 \times 1$ for urine incontinence.

On June 2011 a second MRI was performed showing no significant changes compared to the previous one on May 07, 2010.

\section{Discussion}

We present here a case where autologous ADVF/ADSC have been intravenously administered to a patient moderate AD. The patient displayed a small transient improvement, mainly on her behavioral problems which was verified by standardized test and observations of her family. It has been reported that in $\mathrm{AD}$ experimental models stem cell therapy can be beneficial. ${ }^{7}$

In our case a transient and marginally mild improvement of MMSE and NPI has been recorded (Table 1). That means that cognitive abilities were better and behavioral problems were decreased. Therefore it can be suggested that intravenous autologous stem cell therapy may be beneficial for $\mathrm{AD}$ treatment and justify the setting of a clinical trial. In a setting of a considerable clinical trial larger doses of autologous ADSCs and a repetitive infusion every 1-2 months may be considered. On the other hand the rapid deterioration of the patient's condition following the initial improvement poses questions regarding the long-term effectiveness of stem cell therapy and its possible negative effects/outcomes. These considerations should be explored in detail and clarified in future large scale trials.

Mesenchymal and other bone marrow-derived stem cells are capable of passing the blood-brain barrier and populate the entire central nervous system, differentiating mainly in microglial cells. ${ }^{8}$ In the case of AD, these migrated stem cells can play a neuroprotective role. ${ }^{9}$ It has been recently reported that these cells attenuate amyloid $\beta$-induced damage, inhibit neuronal cell death, ${ }^{4}$ reduce amyloid $\beta$ disposition and induce microglia in an AD experimental model. ${ }^{10}$ Bone marrowderived mesenchymal stem cells reduce brain amyloid $\beta$ deposition and accelerate the activation of microglia in an acutely induced AD mouse model. Accordingly the future of stem cell therapy in $\mathrm{AD}$ is considered promising. Efforts for creating a therapy for $\mathrm{AD}$ using stem cell technologies and their clinical applications are expected to increase in the next years. ${ }^{8}$ However no clinical cases have been reported up-to-date considering the application of stem cell therapies in AD.

Recent reports indicate that ADSCs, which can home into damaged brain areas, are advantageous for the recovery of brain injury in comparison to bone marrow stem cells. ${ }^{11}$ ADSCs have been extensively used in preclinical and clinical studies. ${ }^{12}$ In the clinical setting two main preparations containing stem cells from adipose tissue have been used, that have been recently named "adipose-derived stromal cells" and "adipose-derived vascular fraction". 13 "Adiposederived vascular fraction" also named "Non-expanded adipose stromal vascular fraction" (ADVF/ADSC), is the simplest form to be used in an autologous setting, because its preparation requires minimum manipulation and has been shown to be safe, if the concerns of embolism and sepsis can be controlled. ${ }^{13}$

ADVF/ADSCs have been first isolated by Rodbell and Jones $^{4}$ using collagenase ${ }^{12}$ and have been used in preclinical and clinical trial settings over the whole spectrum of regenerative medicine from simple fat transfer to tissue engineering. According to recent views "the intravenous systemic administration of ADVF/ADSC provides an easy 
and minimally invasive treatment modality". Safety of intravenous ADVF/ADSC administration has been recently shown. ${ }^{14}$ This approach has been also used in a series of preclinical and clinical investigations with no adverse effects indicated up to now. ${ }^{13,15,16}$

Recent data suggest that ADVF/ADSC can be used as a novel valuable therapeutic tool for chronic inflammatory diseases of the central nervous system. ${ }^{17}$ Several recent investigators report that ADVF/ADSC can be used in cellular therapies and provide a functional benefit in a wide range of neurological diseases. ${ }^{18}$ Intravenous administration of ADVF/ADSC for the treatment of neurological disease has been successfully investigated in animal models of stroke, autoimmune encephalitis and olfactory dysfunction. ${ }^{13}$ However, no translating efforts have been published up to date. We report here the first, according to our knowledge, case a patients with $\mathrm{AD}$ who were administered intravenously ADVF/ADSC for the treatment of moderate AD which we hope will serve as a basis for further research of the possibilities opened up by ADVF/ADSC. It should be noted that despite the large amount of data concerning the mechanism of action of stem cells such data derive from preclinical studies. Further studies are needed in order to assess and describe the mechanism of action of stem cells in the human brain of patients with neurodegenerative diseases.

\section{Conclusion}

We noticed and recorded a transient mild improvement in this patient 1 month after the intervention but this improvement was not stable.

Some observations and avenues of further research follow:

1. The intervention is effective but it could be more effective if the stem cell administration is repeated every month. There is a study with animals which support also this strategy. ${ }^{14}$

2. Another issue is the cells' concentration that could affect the therapeutic result. Therefore, we could say that there is a possibility of amount-based phenomena.

3. Maybe, the mesenchymal stem cells (MSC) injection should be performed at the early stages of $\mathrm{AD}$ in order to achieve better results.

4. Human MSCs secrete neurotrophins such as brain-derived neurotrophic factor which can contribute to anatomical and functional recovery of the brain. Brain-derived neurotrophic factor can affect neuronal excitability and synaptic transmission. Maybe this action can only be effective in early stages when a significant amount of gray matter cells and synapses still exist.

\section{Disclosure}

The authors report no conflicts of interest in this work.

\section{References}

1. Alzheimer's Association. 2009 Alzheimer's disease facts and figures. Alzheimers Dement. 2009;5(3):234-270.

2. Alzheimer Disease International. World Alzheimer Report 2014, Dementia and Risk Reduction: An analysis of protective and modifiable factors. 2014. Available from: https://www.alz.co.uk/research/ WorldAlzheimerReport2014.pdf. Accessed September 23, 2015.

3. Lalu MM, McIntyre L, Pugliese C, et al; Canadian Critical Care Trials Group. Safety of cell therapy with mesenchymal stromal cells (SafeCell): a systematic review and meta-analysis of clinical trials. PLoS One. 2012;7(10):e47559.

4. Rodbell M, Jones AB. Metabolism of isolated fat cells. 3. The similar inhibitory action of phospholipase $\mathrm{C}$ (Clostridium perfringens alpha toxin) and of insulin on lipolysis stimulated by lipolytic hormones and theophylline. J Biol Chem. 1966;241(1):140-142.

5. Tzouvelekis A, Koliakos G, Ntolios P, et al. Stem cell therapy for idiopathic pulmonary fibrosis: a protocol proposal. J Transl Med. 2011;9:182.

6. Mitchell JB, McIntosh K, Zvonic S, et al. Immunophenotype of human adipose-derived cells: temporal changes in stromal-associated and stem cell-associated markers. Stem Cells. 2006;24(2):376-385.

7. Neirinckx V, Coste C, Rogister B, Wislet-Gendebien S. Concise review: adult mesenchymal stem cells, adult neural crest stem cells, and therapy of neurological pathologies: a state of play. Stem Cells Transl Med. 2013;2(4):284-296.

8. Simard AR, Rivest S. Bone marrow stem cells have the ability to populate the entire central nervous system into fully differentiated parenchymal microglia. FASEB J. 2004;18(9):998-1000.

9. Simard AR, Rivest S. Neuroprotective properties of the innate immune system and bone marrow stem cells in Alzheimer's disease. $\mathrm{Mol}$ Psychiatry. 2006;11(4):327-335.

10. Malm TM, Koistinaho M, Pärepalo M, et al. Bone-marrow-derived cells contribute to the recruitment of microglial cells in response to beta-amyloid deposition in APP/PS1 double transgenic Alzheimer mice. Neurobiol Dis. 2005;18(1):134-142.

11. Ikegame Y, Yamashita K, Hayashi Si, et al. Comparison of mesenchymal stem cells from adipose tissue and bone marrow for ischemic stroke therapy. Cytotherapy. 2011;13(6):675-685.

12. Katz A, Mericli A. Stem cells derived from fat. In: Atala A, Lanza R, Thomson JA, Nerem RM, editors. Principles of Regenerative Medicine. San Diego: Elsevier Academic Press; 2011:365-381.

13. Casteilla L, Planat-Benard V, Laharrague P, Cousin B. Adipose-derived stromal cells: their identity and uses in clinical trials, an update. World J Stem Cells. 2011;3(4):25-33.

14. Ra JC, Shin IS, Kim SH, et al. Safety of intravenous infusion of human adipose tissue-derived mesenchymal stem cells in animals and humans. Stem Cells Dev. 2011;20(8):1297-1308.

15. Fang B, Song YP, Li N, Li J, Han Q, Zhao RC. Resolution of refractory chronic autoimmune thrombocytopenic purpura following mesenchymal stem cell transplantation: a case report. Transplant Proc. 2009;41(5):1827-1830.

16. Riordan NH, Ichim TE, Min WP, et al. Non-expanded adipose stromal vascular fraction cell therapy for multiple sclerosis. J Transl Med. 2009;7:29e.

17. Constantin G, Marconi S, Rossi B, et al. Adipose-derived mesenchymal stem cells ameliorate chronic experimental autoimmune encephalomyelitis. Stem Cells. 2009;27(10):2624-2635.

18. Miller RH, Bai L, Lennon DP, Caplan AI. The potential of mesenchymal stem cells for neural repair. Discov Med. 2010;9(46):236-242. 
Journal of Neurorestoratology

\section{Publish your work in this journal}

The Journal of Neurorestoratology is an international, peer-reviewed, open access online journal publishing original research and review articles on the subject of Neurorestoratology. To provide complete coverage of this revolutionary field the Journal of Neurorestoratology will report on relevant experimental research, technological advances, and

clinical achievements. The manuscript management system is completely online and includes a very quick and fair peer-review system, which is all easy to use. Visit http://www.dovepress.com/testimonials.php to read real quotes from published authors.

Submit your manuscript here: http://www.dovepress.com/journal-of-neurorestoratology-journal 